\title{
Grid based Cooperative Spectrum Sensing in Cognitive Networks under Correlated Shadowing
}

\author{
Xia Li, Petri Mähönen \\ Institute for Networked Systems, RWTH Aachen University \\ Kackertstrasse 9, D-52072 Aachen, Germany \\ Email:\{xia,pma\}@inets.rwth-aachen.de
}

\begin{abstract}
Correlated shadowing can have significant effects on the performance of cooperative spectrum sensing. In this paper, we investigate a grid based cooperative spectrum sensing in cognitive networks under correlated shadowing. We study three types of grids: equilateral triangular, square and hexagonal grids, where the sensing users are deployed at the intersections of the polygons. The collaboration is formed as a location testing problem with Gaussian noise, where the shadow fading is modeled as a multivariate Gaussian process with two dimensional exponential correlation model. We have evaluated the results regards to the number of collaborating users combined with the separation distance of two adjacent users. It is found that the three layouts yield the same detection performance as that of experiencing independent shadowing when the separation distance exceeds approximately 6 times the decorrelation distance in urban areas.
\end{abstract}

\section{INTRODUCTION}

Cognitive Radio has been widely regarded as the technique to increase spectrum utilization in the next generation wireless communication systems through implementing opportunistic spectrum access. A critical component of cognitive radio is spectrum sensing which enables secondary users to detect the absence or presence of licensed transmissions over primary bands and hence make use of the unused spectrum slots. The spectrum sensing itself can be used also to enhance the performance of other existing and future wireless systems, e.g., femtocell based LTE systems have been shown to benefit from the better knowledge of their radio environment.

Many spectrum sensing techniques have been proposed in the literature [1]. A typical technique for detecting the existence of primary transmissions is energy detection. Although the energy detection has some merits, it is susceptible to random variations of the received power due to multipath fading and shadowing. Also, the limited sensitivity of a radio does not allow a secondary user to detect very low power signals. These result in the well known spectrum sensing problems of missed detection and false alarm. In order to eliminate the detection errors, cooperative spectrum sensing has been proposed to counteract the fading effects as well as the limitations of energy detection in the extremely low signal regime. In cooperative schemes, observations of different sensors are processed locally to generate compressed messages which are sent to a fusion center for combinationally making a final decision on the presence or absence of primary signals.

One key challenge of cooperative spectrum sensing is the selection of sensing users. A cooperative scheme is most effective when it can achieve maximum spatial diversity gain with a minimum number of sensing users. Recent studies have shown that the expected benefits of cooperation could be severely hampered when signals sensed by cooperative users experience correlated shadowing [2], [3]. Too many correlated sensors could become a detrimental to the spectrum detection performance due to limited spatial diversity and excessive transmission overhead during information combining. The cooperative spectrum sensing performance can be maximized only when the sensors experience independent shadowing and multipath fading.

Considerable research has been conducted to investigate the impact of correlated shadowing on the cooperative detection and sensing user selection [2]-[10]. The authors in [5] has built a location testing model under correlated shadowing and figured out a lower bound of false alarm probability which is regardless of the growth of number of sensors over a finite area. The work in [8] and [9] focuses on the linear combinations of local observations and studies the power sum of correlated log-normal random variables. In [7], without delving into the detection performance, the sensor selection is analyzed regardless of the optimal number of sensors. The authors assume that the desired number of sensors is fixed and known in advance, and propose methodologies to select sensors which can minimize the total geographic correlation measures. In [3], [6], [10], the authors have studied the number of collaborating users in terms of distance spread. However, they only study one-dimensional distributions where sensors are deployed on a line, which makes the results limited in terms of generality and real world application.

In this paper, we consider two dimensional distributions of collaborating sensors and investigate the cooperative detection performance under distance dependent log-normal correlated shadowing. We studied three types of regular grid: triangle, square and hexagon. The sensors are distributed at the grid intersections. The grid distributions of sensors are not necessarily optimal but can play a crucial role in the prediction and resolution of the spectrum sensing cooperation solutions. Typically, the grid based analysis may be especially useful for some modern cities with straight grid layouts where the sensors could be distributed along the streets.

The shadow fading has been widely approximated by a lognormal distribution in the literature. However, it is noted that, due to the inherent complexity of handling multidimensional 
correlated log-normal random variables, signals sensed by different users over various locations are usually assumed to be log-normal distributed with the same mean and variance, even though shadowing effects over different locations could vary significantly due to different propagation conditions. Little attention has been paid to different shadowing variances. Our work models the shadowing variance over a two dimensional space as a random variable following a uniform distribution. Meaningful results have been obtained.

The rest of the paper is organized as follows. Section II describes the general model and assumptions used in our study. In Section III, we present the grid based cooperative spectrum sensing and derive the detection and false alarm probabilities based on the soft fusion rule. In Section IV, we conduct the numerical results analysis with conclusion given in Section V.

\section{System Model AND Assumptions}

We consider that a secondary network is located far away from the primary transmitter with a distance $r$ and the size of the secondary network is small compared to $r$, so that all secondary users are assumed to experience the same path loss. The secondary network consists of $n$ sensing nodes distributed in a $D \times D$ square area and a fusion center which does not perform sensing. The statistic of received signal power in a logarithmic scale at the $i$ th sensing node is denoted by $\gamma_{i}$ and simply expressed as:

$$
\gamma_{i}(r)=\gamma_{t r}-10 \alpha \lg (r)+\chi_{i},
$$

where $\gamma_{t r}$ is primary transmitter power; $\alpha$ denotes the signal power decay factor; and $\chi_{i}$ represents the shadowing effects with log-normal uncertainty in estimation of the received power. The symbol $\chi_{i}$ denotes a Gaussian random variable with mean zero and variance $\sigma_{i}^{2}(r)$. According to (1), $\gamma_{i}$ follows a Gaussian distribution with mean $\mu(r)=\gamma_{t r}-10 \alpha \lg (r)$ and variance $\sigma_{i}^{2}(r) . \mu(r)$ of all the sensing nodes is assumed to be identical due to the large value of $r$. The standard deviation $\sigma_{i}$ usually varies at different locations in the range between $4 \mathrm{~dB}$ and $13 \mathrm{~dB}$, since different propagation paths may experience different environmental shadowing effects due to various types of obstructions such as hills, buildings and trees. Let $\gamma$ denote a vector containing $\gamma_{i}(1 \leq i \leq n)$. We have the following $n$-dimensional multivariate normal distribution:

$$
\gamma \sim N(\boldsymbol{\mu}(r), \boldsymbol{\Sigma})
$$

where $\boldsymbol{\mu}$ is a $n$-dimensional vector of $\mu(r)$; and $\boldsymbol{\Sigma}$ represents the $n \times n$ covariance matrix of the shadowing effects as follows:

$$
\Sigma_{i j}= \begin{cases}\rho_{i j} \sigma_{i} \sigma_{j}, & i \neq j \\ \sigma_{i}^{2}, & \text { otherwise }\end{cases}
$$

where $\rho_{i j}$ is the correlation coefficient between node $i$ and node $j$. In our model, we adopt the popular Gudmundson correlation model [11] in which $\rho_{i j}=e^{-r_{i j} / D_{\text {corr }}}$, and $r_{i j}$ is the distance between node $i$ and node $j$. The decorrelation distance $D_{\text {corr }}$ depends on the environment. Through fitting of measured data, Gudmundson derived $D_{\text {corr }}$ to be $503.9 \mathrm{~m}$

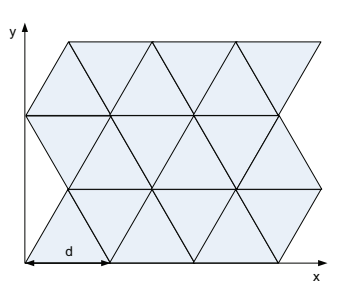

(a) triangular grid

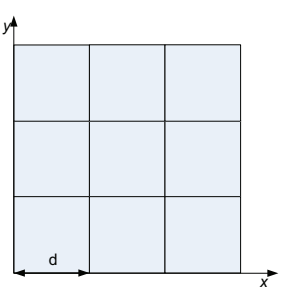

(b) square grid

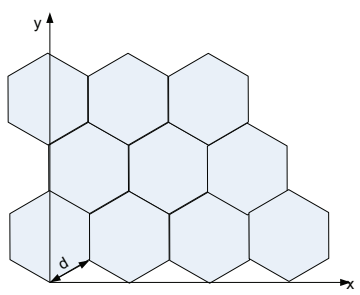

(c) hexagonal grid

Fig. 1. Grid distributions of sensing nodes.

in suburban areas at $900 \mathrm{MHz}$ and $8.3058 \mathrm{~m}$ in urban areas at $1.7 \mathrm{GHz}$

The probability density function (p.d.f) of $\gamma$ is given by:

$$
f_{\gamma}(\hat{\gamma})=\frac{1}{\sqrt{(2 \pi)^{n}|\boldsymbol{\Sigma}|}} \exp \left\{-\frac{1}{2}(\hat{\gamma}-\boldsymbol{\mu})^{T} \boldsymbol{\Sigma}^{-1}(\hat{\gamma}-\boldsymbol{\mu})\right\},
$$

where $|\boldsymbol{\Sigma}|$ and $\boldsymbol{\Sigma}^{-1}$ are the determinant and inverse of $\boldsymbol{\Sigma}$, respectively.

The binary hypotheses detection problem can be formed as a location testing problem with Gaussian noise. Denote the distance $R$ as a safe distance from the primary transmitter beyond which unlicensed transmissions statistically do not generate harmful interference to the licensed users. Thus, the spectrum sensing is reduced to question to determine whether the mean received power at a certain location is higher than the power expected at the edge of the protection zone defined by $R$. Hence we have:

$$
\begin{aligned}
& H_{0}: \gamma \sim N(\boldsymbol{\mu}(R+\varepsilon), \boldsymbol{\Sigma}), \\
& H_{1}: \gamma \sim N(\boldsymbol{\mu}(R), \boldsymbol{\Sigma}),
\end{aligned}
$$

where $\varepsilon$ specifies how far the node is located outside the range defined by $R . H_{0}$ and $H_{1}$ denote the absence and presence of primary signals on the channel respectively.

\section{GRID BASEd Cooperative SENSING}

\section{A. Grid based Sensor Deployment}

We consider three types of grid distributions as shown in Fig. 1: equilateral triangular, square and hexagonal grids, which are the only three regular polygons to completely fill a Euclidean plane without any gaps and overlaps. The sensing nodes are distributed at the corners of the polygons. Let $d$ denote the separation distance from one sensing node to its most adjacent nodes. For any sensing node located away from the edge, the number of nearest neighbors for three types of distributions is different: 6 for triangle, 4 for square and 3 
for hexagon. It is clear that the triangular distribution has the highest sensor density. The number of sensors in a $D \times D$ square for three grid distributions are derived as follows:

$$
\begin{aligned}
& n_{t}=\left\{\begin{array}{lr}
\left\lceil\frac{2 D}{\sqrt{3} d}\right\rceil *\left\lceil\frac{D}{d}\right\rceil, & \operatorname{rem}\left(\frac{D}{d}\right) \in\left[\frac{1}{2}, 1\right) \\
\left\lceil\frac{D}{d}\right\rceil *\left\lceil\frac{D}{\sqrt{3} d}\right\rceil+\left(\left\lceil\frac{D}{d}\right\rceil-1\right)\left\lceil\frac{D}{\sqrt{3} d}-\frac{1}{2}\right\rceil, & \text { otherwise }
\end{array}\right. \\
& n_{s}=\left\lceil\frac{D}{d}\right\rceil^{2}, \\
& n_{h}=\left\{\begin{array}{lr}
2\left\lceil\frac{2 D}{\sqrt{3} d}\right\rceil *\left\lceil\frac{D}{3 d}\right\rceil, & \operatorname{rem}\left(\frac{D}{3 d}\right) \in\left[\frac{2}{3}, 1\right) \\
2\left\lceil\frac{2 D}{\sqrt{3} d}\right\rceil *\left\lceil\frac{D}{3 d}\right\rceil-\left\lceil\frac{D}{\sqrt{3} d}\right\rceil, & \operatorname{rem}\left(\frac{D}{3 d}\right) \in\left[\frac{1}{2}, \frac{2}{3}\right) \\
2\left\lceil\frac{2 D}{\sqrt{3} d}\right\rceil *\left\lceil\frac{D}{3 d}\right\rceil-\left(\left\lceil\frac{D}{\sqrt{3} d}\right\rceil+\left\lceil\frac{D}{\sqrt{3} d}-\frac{1}{2}\right\rceil\right), \\
2\left\lceil\frac{2 D}{\sqrt{3} d}\right\rceil *\left\lceil\frac{D}{3 d}\right\rceil-\left(\left\lceil\frac{D}{\sqrt{3} d}\right\rceil+2\left\lceil\frac{D}{\sqrt{3} d}-\frac{1}{2}\right\rceil\right),
\end{array}\right. \\
& \operatorname{rem}\left(\frac{D}{3 d}\right) \in\left[0, \frac{1}{6}\right)
\end{aligned}
$$

where $\lceil a\rceil$ rounds the $a$ to the nearest integer greater than $a ; \operatorname{rem}\left(\frac{a}{b}\right)$ is the remainder of $a / b . n_{t}, n_{s}$ and $n_{h}$ denote the number of sensors for triangular, square and hexagonal distributions respectively. The relationship of them with the separation distance $d$ is shown in Fig. 2.

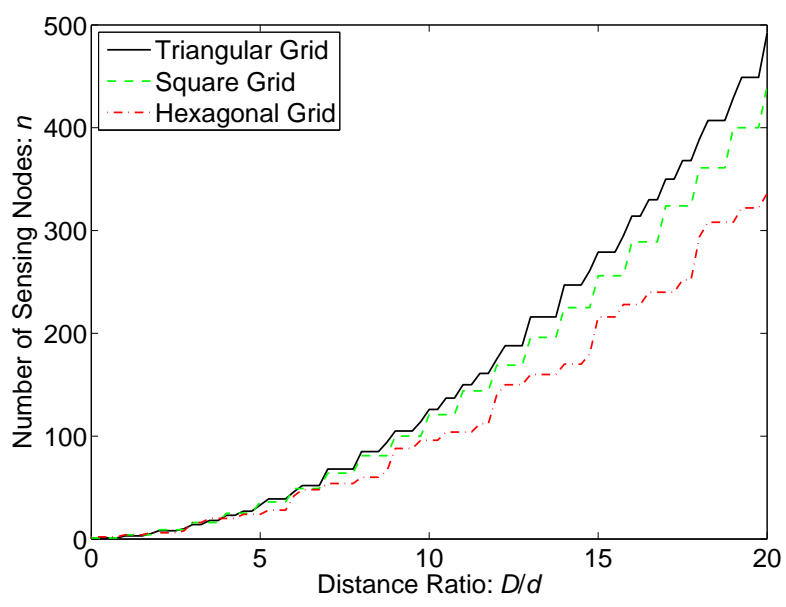

Fig. 2. Number of sensing nodes in three grids

\section{B. Soft Decision Fusion Strategy}

In soft decision, each cooperative sensor sends the statistics of its local observation to the fusion center which uses a Square-Law Combing mechanism to combine multiple observations and make a final decision on the state of the primary channel. The cooperative spectrum detection is given by the Neyman Pearson Lemma:

$$
\Lambda(\gamma)=\frac{f\left(\gamma \mid H_{1}\right)}{f\left(\gamma \mid H_{0}\right)} \underset{H_{0}}{\stackrel{H_{1}}{\gtrless}} \lambda,
$$

where $\Lambda(\gamma)$ is the likelihood ratio of the observations from $n$ sensing users. $f\left(\gamma \mid H_{i}\right)$ is the p.d.f. of the received power given by (4). Hence, $\Lambda(\gamma)$ is derived as:

$$
\Lambda(\gamma)=\exp \left\{\boldsymbol{\Delta}^{T} \boldsymbol{\Sigma}^{-1}\left(\gamma-\frac{\boldsymbol{\mu}_{\mathbf{1}}+\boldsymbol{\mu}_{\mathbf{0}}}{2}\right)\right\},
$$

where $\boldsymbol{\mu}_{\mathbf{1}}=\boldsymbol{\mu}(R), \boldsymbol{\mu}_{\mathbf{0}}=\boldsymbol{\mu}(R+\varepsilon)$, and $\boldsymbol{\Delta}=\boldsymbol{\mu}_{\mathbf{1}}-\boldsymbol{\mu}_{\mathbf{0}}$. The detection and false alarm probabilities are further derived as [12]:

$$
\begin{aligned}
& P_{D}=Q\left(\frac{\hat{\lambda}-\mu_{D}}{\omega}\right), \\
& P_{F}=Q\left(\frac{\hat{\lambda}-\mu_{F}}{\omega}\right),
\end{aligned}
$$

where $\hat{\lambda}=\ln \lambda+\frac{1}{2} \boldsymbol{\Delta}^{T} \boldsymbol{\Sigma}^{-1}\left(\boldsymbol{\mu}_{\mathbf{1}}+\boldsymbol{\mu}_{\mathbf{0}}\right) ; \mu_{D}=\boldsymbol{\Delta}^{T} \boldsymbol{\Sigma}^{-1} \boldsymbol{\mu}_{\mathbf{1}}$; $\mu_{F}=\boldsymbol{\Delta}^{T} \boldsymbol{\Sigma}^{-1} \boldsymbol{\mu}_{\mathbf{0}}$; and $\omega=\sqrt{\boldsymbol{\Delta}^{T} \boldsymbol{\Sigma}^{-1} \boldsymbol{\Delta}} ; Q(x)$ is the complementary distribution function of a standard Gaussian variable and is given by $\frac{1}{\sqrt{2 \pi}} \int_{x}^{\infty} e^{-t^{2} / 2} d t$.

The relationship between $P_{D}$ and $P_{F}$ becomes:

$$
Q^{-1}\left(P_{F}\right)-Q^{-1}\left(P_{D}\right)=\sqrt{\boldsymbol{\Delta}^{T} \boldsymbol{\Sigma}^{-1} \boldsymbol{\Delta}},
$$

where $\omega=\sqrt{\Delta^{T} \boldsymbol{\Sigma}^{-1} \boldsymbol{\Delta}}$ can be interpreted as a measure of signal-to-noise ratio.

Since all sensing nodes form only a small sized network with the very large separation distance $R$ from the primary transmitter, we can make an assumption that the mean $\mu_{0}=\mu(R+\varepsilon)$ is the same for all the nodes as the small variance of $\varepsilon$ can be ignored compared to $R$. The quantity $\omega$ becomes $\Delta \sqrt{\mathbf{1}^{T} \boldsymbol{\Sigma}^{-1} \mathbf{1}}$, where $\mathbf{1}$ is a $n$-dimensional vector of ones. Given a fixed $P_{F}, P_{D}$ increases monotonically as the separation in means $\Delta$ and the quantity $\psi(n, d)=\mathbf{1}^{T} \boldsymbol{\Sigma}^{-1} \mathbf{1}$. According to (1), $\Delta$ is given by $\Delta=\alpha \lg \frac{R+\varepsilon}{R}$ and is independent of the distributions of sensors. $\psi(n, d)$, which is the same as the derivation in [5], reflects the diversity order of cooperative spectrum sensing. As a result, the performance of optimum cooperative detection in lognormal noise can be improved by increasing $\psi(n, d)$ through adjusting the separation distance $d$. An optimum number of sensors $n$ can then be obtained corresponding to the optimal $d$.

\section{NumERICAL RESUlts}

In this section, numerical results are provided based on the analysis of previous sections. We compute false alarm probability $P_{F}$ given a predefined detection probability $P_{D}=$ 0.95, and investigate how $P_{F}$ varies with the separation distance $d$ and the number of sensors $n$. In the calculation, $D_{\text {corr }}=8.3058, \alpha=3.5$, and $\frac{\varepsilon}{R}=0.01$ are used. The shadowing variance $\sigma_{k}$ of each sensor is different and uniformly distributed over a range $[a, b]$. The obtained results are averaged over 10000 different realizations of shadowing variances through the Monte-Carlo approach.

To analyze the detection performance of grid based cooperative sensing, the first task is to define and obtain the $n$ dimensional covariance matrix $\boldsymbol{\Sigma}$. Let the set of secondary users indices given by $(1, \cdots, k, \cdots, n)$, and $(l, m)$ be the row and column indices of one secondary user in the Cartesian coordinate system as shown in Fig. 1. We define $k$ as $k(l, m)=\sum_{i=1}^{l} l_{i}+m$, where $l_{i}$ denotes the number 


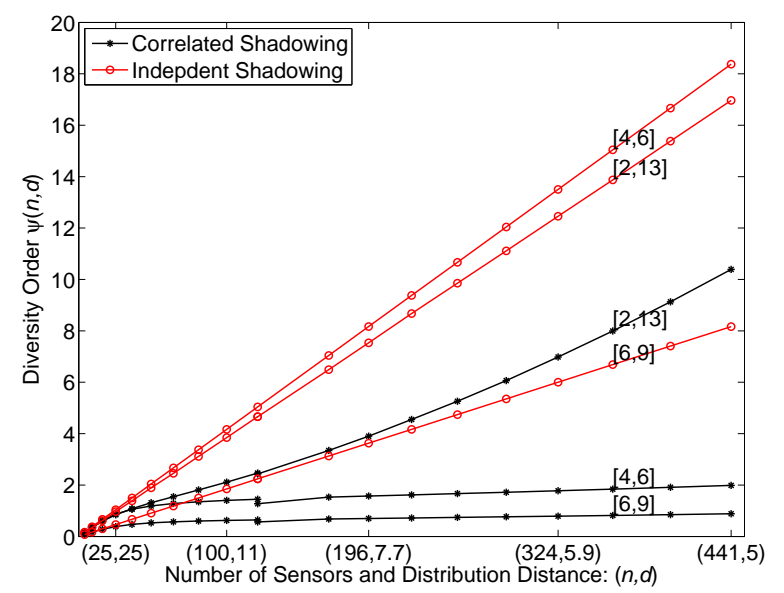

(a)

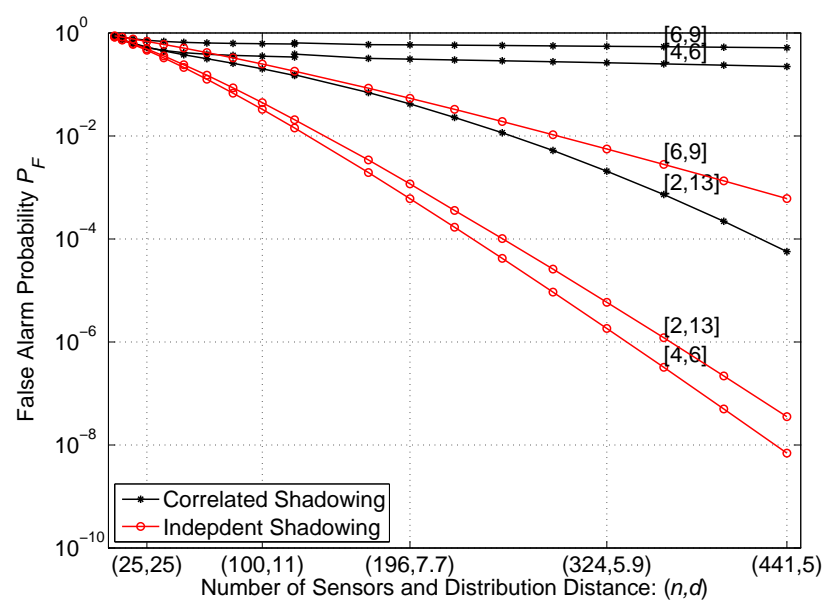

(b)

Fig. 3. Detection performance of square grid with $D=100 \mathrm{~m}$

of sensors on $i$ th row. Correspondingly, we can find the coordinates for each secondary user and hence obtain the 2dimensional correlation coefficient $\rho_{i j}$ between node $i$ and node $j: \rho_{i j}=\exp \left(-\frac{\sqrt{\left(x_{i}-x_{j}\right)^{2}+\left(y_{i}-y_{j}\right)^{2}}}{D_{\text {corr }}}\right)$.

Fig. 3 shows the detection performance of square grid distributions with $D=100 \mathrm{~m}$. In the figure, it is clear that the false alarm probability $P_{F}$ is inversely proportional to the the quantity $\psi(n, d)=\mathbf{1}^{T} \boldsymbol{\Sigma}^{-1} \mathbf{1}$. We vary $D / d$ from 1 to 20 at the step of one to evaluate the impact of separation distance $d$. For each given $d$, there is a corresponding number of sensors $n$ as shown in Fig. 2. The x-axis in Fig. 3 stands for the combination of $(n, d)$. It can be seen that the detection performance is improved as $d$ decreases, but at the cost of increasing $n$.

The shadowing effects on the detection performance are also examined in Fig. 3. For each run of computation, the lognormal shadowing variance at each sensor location follows a uniform distribution over the range $[a, b]$. A great number of simulations have been run with different sets of $[a, b]$. Through observations, it is found that the optimal detection performance is likely to be obtained with a small value of $a$, even though the uniform distributions have the same average. For example, in the figure, $[2,13]$ and $[6,9]$ have the same mean 7.5 and different variances. However, the detection over correlated shadowing of the former even outperforms that over independent shadowing of the latter. Hence, we deduce that, among a group of sensors, those located at sites experiencing small shadowing effects contribute dominantly to the cooperative spectrum sensing. In the figure, we also have one interesting finding. Note that under independent and identically distributed (i.i.d.) shadowing, $\psi(n, d)$ can be expressed as:

$$
\psi(n, d)=\sum_{i=1}^{n} \frac{1}{\sigma_{i}^{2}}
$$

The average of $\psi(n, d)$ over the interval $[a, b]$ is given by:

$$
E(\psi(n, d))=\frac{n}{a b},
$$

We can see that, for cooperative sensors which experience independent shadowing, the detection performance increases monotonically as the product of $a$ and $b$.

Fig. 4 shows the comparison of detection performance for three types of grids. The square grid slightly outperforms the triangular grid when the value of $D / d$ is less than 6 (i.e., smaller number of sensing nodes). The triangular grid has the best performance as the separation distance $d$ continues to decrease. However, this performance enhancement is achieved at the cost of more sensors.

In Fig. 5, the number of sensing nodes is fixed at $n=16$ for three grids. The separation distance $d$ is varied. The shadowing variance of one node is taken from $[2,13]$. It can be seen that, the false alarm probabilities of three layouts are different for small $d$ and eventually converge to the same value with the increase of $d$. The three layouts start having the same performance at around 6 times the decorrelation distance when the correlation coefficient $\rho$ becomes around 0.0025. This observation is also reflected by the average correlation coefficients obtained in Fig. 5(b) where the coefficients tend to zero when the separation distance meets the condition $d \geq 6 D_{\text {corr }}$, which represents independent shadowing effects. Also, it is observed that among three distributions, the hexagonal grid has the best performance. This is because the hexagonal grid has a less dense distribution than the other two grids, and hence is less affected by the shadow correlation.

\section{CONCLUSIONS}

In this paper we formulate the cooperative spectrum sensing over correlated log-normal shadowing as a location testing problem, and compare the detection performance for three types of regular grid distributions of collaborative sensing users. Different shadowing variances across various locations 


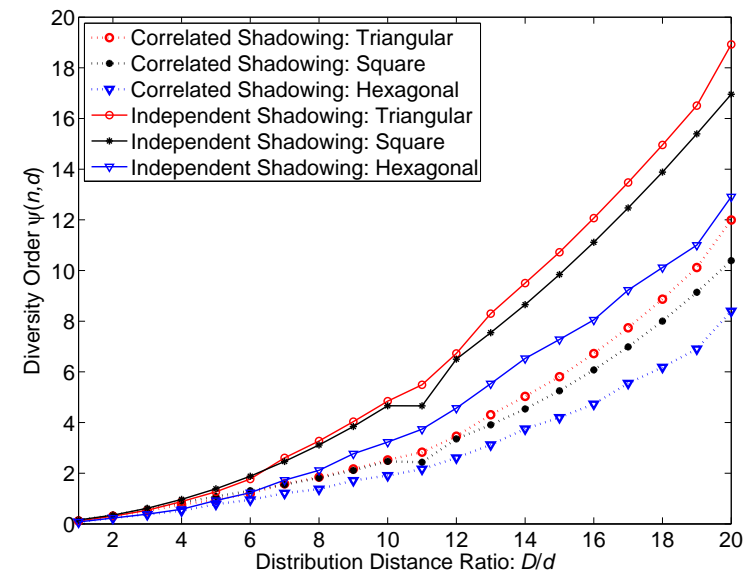

(a)

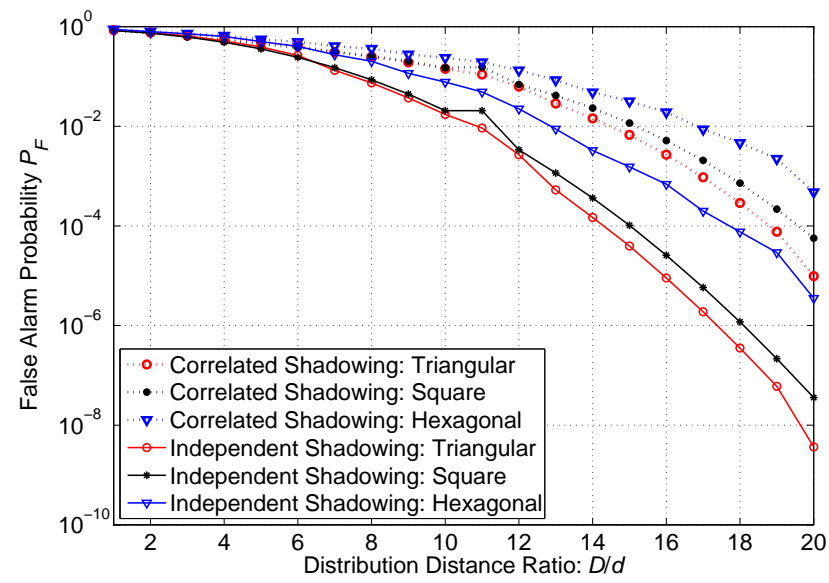

(b)

Fig. 4. Comparison of detection performance for three grids with $D=100 \mathrm{~m}$

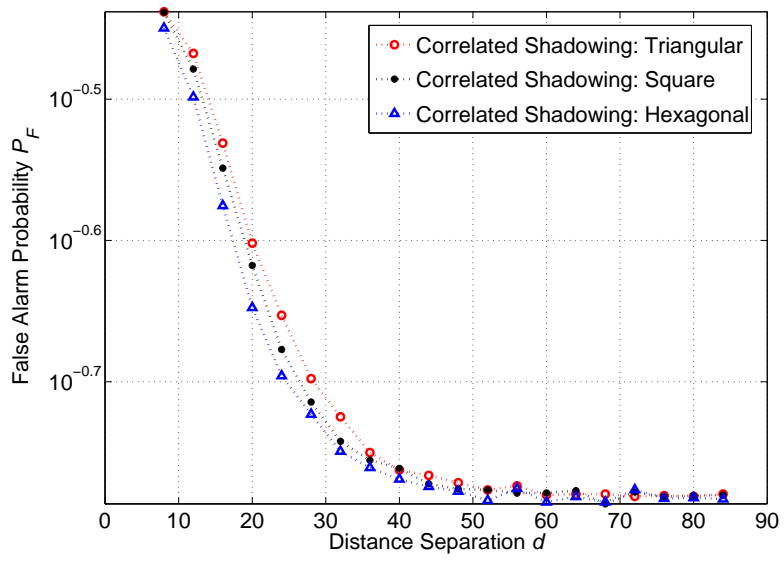

(a)

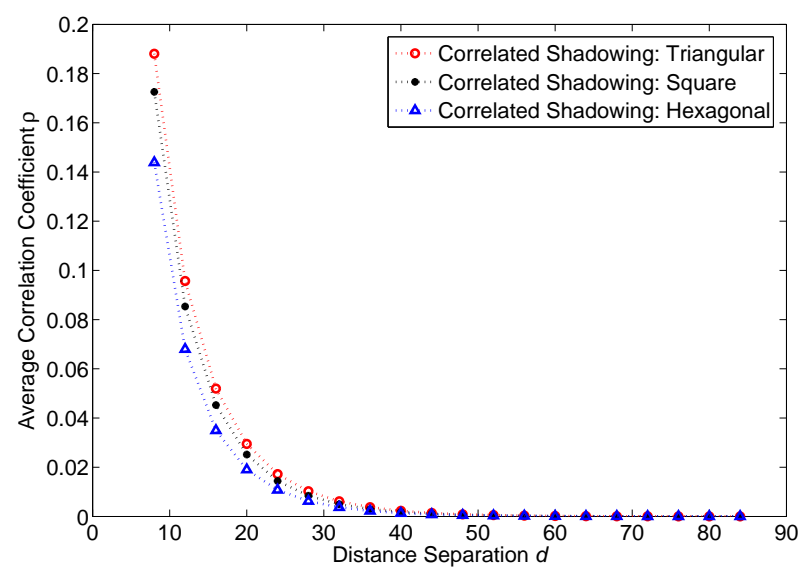

(b)

Fig. 5. Comparison of detection performance for three grids with $n=16$

have been taken into account in our study and the values of variances are drawn from uniform distributions. Our results show that among a group of collaborative sensing users, the sensors experiencing the least shadowing effects contribute dominantly to the cooperative detection performance, which means decisions made by sensing users with high detection SNRs are more reliable. Moreover, when the separation distance exceeds approximately 6 times the Gudmundson decorrelation distance, the three layouts yield the same detection performance as that of experiencing independent shadowing. It is found that hexagonal grid deployment behaves the best due to its sparse distribution compared to the other two types of grids. Our study has practical implications as the grid based analysis can provide some insight into the required resolution of cooperative spectrum sensing in terms of the user selection and deployment. The application of our results is limited in the sense that the real world shadow fading map may not be simply modeled using uniform distributions [13]. Including more realistic shadowing correlation model could be part of our future work.

\section{ACKNOWLEDGMENT}

We acknowledge a partial financial support from European Union through EU FP7 project INFSO-ICT-248303 QUASAR. We also thank the support from DFG through UMIC research center.

\section{REFERENCES}

[1] D. Noguet et al., "Sensing techniques for cognitive radio- state of the art and trends," http://grouper.ieee.org/groups/scc41/6/documents/ whitepapers/P1900.6_WhitePaper_Sensing_final.pdf, 2009.

[2] A. Ghasemi and E. S. Sousa, "Collaborative spectrum sensing for opportunistic access in fading environments," in Proceedings of IEEE Dyspan, Nov. 2005, pp. 131-136.

[3] A. Ghasemi and E. Sousa, "Asymptotic performance of collaborative spectrum sensing under correlated log-normal shadowing," IEEE Communication Letter, vol. 11, no. 1, pp. 34-36, Jan. 2007.

[4] I. Akyildiz, B. Lo, and R. Balakrishnan, "Cooperative spectrum sensing in cognitive radio networks: A survey," Physical Communication (Elsevier) Journal, vol. 4, no. 1, pp. 44-62, Mar. 2011. 
[5] E. Visotsky, S. Kuffner, and R. Peterson, "On collaborative detection of tv transmissions in support of dynamic spectrum sensing," in Proceedings of IEEE Dyspan, November 2005, pp. 338-345.

[6] Y. Chen, "Analysis of user selection in collaborative spectrum sensing with correlated shadowing," Wireless Communications and Mobile Computing, Feb. 2011.

[7] Y. Seln, H. Tullberg, and J. Kronander, "Sensor selection for cooperative spectrum sensing," in Proceedings of IEEE Dyspan, 2008, pp. 1-11.

[8] M. Di Renzo, F. Graziosi, and F. Santucci, "Cooperative spectrum sensing in cognitive radio networks over correlated log-normal shadowing," in Proceedings of IEEE VTC, Apr. 2009.

[9] N. Reisi, V. Jamali, M. Ahmadian, and S. Salari, "Cooperative spectrum sensing over correlated log-normal channels in cognitive radio networks based on clustering," in Proceedings of IEEE ConTel, 2011, pp. 161168.

[10] S. M. Mishra, A. Sahai, and R. W. Brodersen, "Cooperative sensing among cogntiive radios," in Proceedings of IEEE ICC, 2006.

[11] M. Gudmundson, "Correlation model for shadow fading in mobile radio systems," Electronics Letters, vol. 27, pp. 2145-2146, Nov. 1991.

[12] H. Poor, An Introduction to Signal Detection and Estimation, second edition. New York: Springer Verlag, 1994.

[13] I. Forkel, M. Schinnenburg, and M. Ang, "Generation of twodimensional correlated shadowing for mobile radio network simulation," in Proceedings of WPMC, Sept. 2004. 